\title{
Detection in two hospitals of transferable ceftazidime- avibactam resistance in Klebsiella pneumoniae due to a novel VEB $\beta$-lactamase variant with a Lys234Arg substitution, Greece, 2019
}

E. Voulgari ${ }^{1}$, S.D. Kotsakis ${ }^{1}$, P. Giannopoulou ${ }^{2}$, E. Perivolioti ${ }^{3}$, L.S. Tzouvelekis ${ }^{1,4}$, V. Miriagou $^{1}$

1. Laboratory of Bacteriology, Hellenic Pasteur Institute, Athens, Greece

2. Department of Clinical Microbiology, Thriassio General Hospital, Elefsina, Greece

3. Department of Clinical Microbiology, Evangelismos General Hospital, Athens, Greece

4. Department of Microbiology, Medical School, University of Athens, Athens, Greece

Correspondence: Vivi Miriagou (miriagou@pasteur.gr)

Citation style for this article:

Voulgari E., Kotsakis S.D., Giannopoulou P., Perivolioti E., Tzouvelekis L.S., Miriagou V.. Detection in two hospitals of transferable ceftazidime-avibactam resistance in Klebsiella pneumoniae due to a novel VEB $\beta$-lactamase variant with a Lys234Arg substitution, Greece, 2019. Euro Surveill. $2020 ; 25(2): p i i=1900766$. https://doi.org/10.2807/1560-7917.ES.2020.25.2.1900766

Article submitted on 27 Dec 2019 / accepted on 16 Jan 2020 / published on 16 Jan 2020

Two ceftazidime-avibactam (CAZ-AVI)-resistant Klebsiella pneumoniae carbapenemase (KPC)-positive $K$. pneumoniae strains, including one pandrug resistant, were isolated in 2019 from two Greek hospitals. The strains were sequence types (ST)s 258 and 147 and both harboured similar self-transmissible IncA/C2 plasmids encoding a novel Lys234Arg variant of the Vietnamese extended-spectrum $\beta$-lactamase (VEB)-1, not inhibited by AVI (VEB-25). Conjugal transfer of VEB-25-encoding plasmids to Escherichia coli yielded CAZ-AVI-resistant clones, supporting that VEB-25 is directly linked to the derived phenotype.

Ceftazidime-avibactam (CAZ-AVI) is a combination medication, which is active against various multidrugresistant Gram-negative bacteria including Klebsiella pneumoniae carbapenemase (KPC)-K. pneumoniae (Kp) [1]. CAZ-AVI resistance among KPC-Kp strains is still rare and commonly associated with selection of KPC-2/3-derived mutant $\beta$-lactamases, resisting inhibition by avibactam [2-5]. In 2019, in view of the rapid risk assessment raising awareness on the emergence of resistance to ceftazidime-avibactam in carbapenemresistant Enterobacteriaceae in Europe issued by the European Centre for Disease Control and Prevention (ECDC) [6], KPC-Kp isolates referred to Hellenic Pasteur Institute from Greek hospitals were further investigated. CAZ-AVI resistance was confirmed in two of 118 isolates analysed during 2019 and was attributed to the production of a novel Vietnamese extended-spectrum $\beta$-lactamase (VEB)-type. Herein, we describe the characteristics of the isolates possessing this resistance mechanism.
Isolation of ceftazidime-avibactam-resistant strains and antibiotic susceptibility

The first CAZ-AVI resistant KPC-Kp strain (T-970/19) was isolated from blood cultures obtained from a female patient in her 6os hospitalised in the intensive care unit (ICU) of Hospital A. The patient was transferred in July 2019 from another hospital following a prolonged complicated hospitalisation course due to cardiopulmonary arrest and acute respiratory distress syndrome. On Day 10 of hospitalisation, a central line-associated bloodstream infection was identified whereupon blood cultures yielded an isolate (T-970/19) that exhibited an extensively drug-resistant phenotype retaining solely intermediate susceptibility to tigecycline. The second CAZ-AVI-resistant KPC-Kp isolate (E-1037/19) was retrieved from a male patient in his 30 s admitted in August 2019 to the ICU of Hospital B with an epidural haematoma following a traffic accident. The E-1037/19 isolate was obtained on Day 14 of hospitalisation from a bronchoalveolar lavage fluid and exhibited resistance to all clinically available antimicrobials (pandrug resistant). Given the patient's subsequent clinical course and laboratory findings this was considered as a colonisation. None of the patients had received CAZAVI before the isolation of the resistant strains.

\section{Whole genome sequencing and analysis}

Genomic DNA was sequenced on a S5-Ion System platform. In silico multilocus sequence typing (MLST) and capsular polysaccharide (cps)-typing assigned T-970/19 to sequence type (ST)147 (wzi64) and $\mathrm{E}-1037 / 19$ to ST258 clade I (wzi154). Resistance genes were identified using the nucleotide-nucleotide Basic Local Alignment Search Tool (blastn) programme of the BLAST+package and the gene sequences in the Bacterial Antimicrobial Resistance Reference Gene 


\section{TABLE 1}

Antibiotic resistance genes of Vietnamese extended-spectrum $\beta$-lactamase (VEB)-producing Klebsiella pneumoniae clinical strains and Escherichia coli transconjugant clones

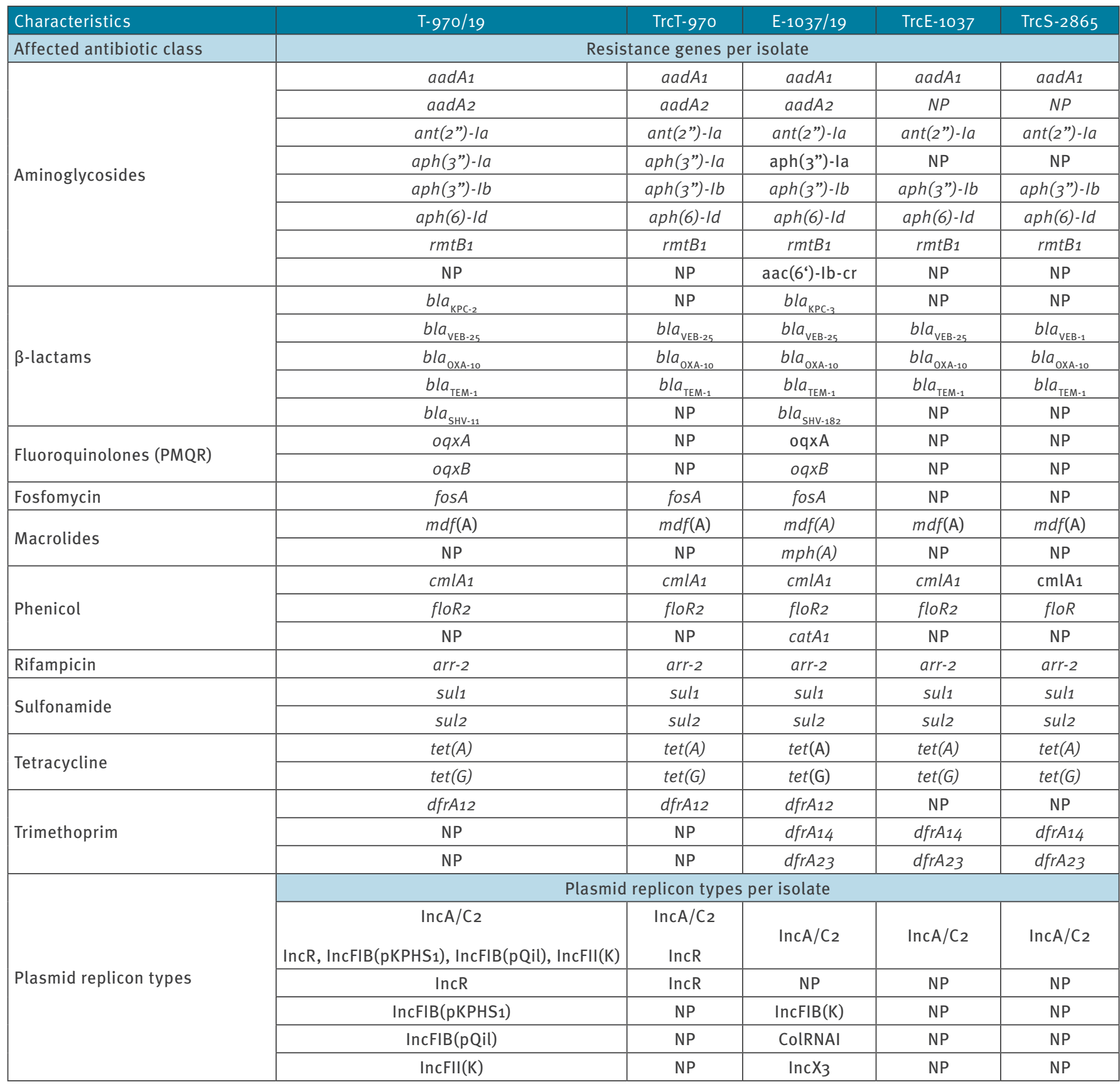

NP: Not present; KPC: Klebsiella pneumoniae carbapenemase; OXA: oxacillinase; PMQR: plasmid-mediated quinolone resistance; SHV: sulfhydryl reagent variable $\beta$-lactamase; TEM: Temoniera $\beta$-lactamase; Trc: transconjugant; VEB: Vietnamese extended-spectrum $\beta$-lactamase.

T-970/19 and E-1037/19 are the VEB-producing Klebsiella pneumoniae clinical strains detected in this study. TrcT-970 and TrcE-1037 are the Escherichia coli transconjugant clones obtained by mating experiments using T-970/19 and E-1037/19 as donors and a $\beta$-lactamsusceptible $E$. coli K12 strain (26R793) as recipient.TrcS-2865 is a ceftazidime-avibactam-susceptible E. coli $26 \mathrm{R} 793$ transconjugant containing $b a_{\mathrm{VEB}-1}$, bla $a_{\mathrm{OXA}-10}$ and bla $a_{\mathrm{TEM}-1}$ that was used for control purposes. 
$\beta$-lactamase content and susceptibility to selected $\beta$-lactam antibiotics of Vietnamese extended-spectrum $\beta$-lactamase (VEB)-producing Klebsiella pneumoniae clinical strains and Escherichia coli transconjugant clones

\begin{tabular}{|c|c|c|c|c|c|c|c|c|}
\hline \multirow[b]{2}{*}{ Isolates } & \multirow[b]{2}{*}{$\begin{array}{l}\beta \text {-lactamase } \\
\text { content }\end{array}$} & \multicolumn{7}{|c|}{ Etest MICs $(\mu \mathrm{g} / \mathrm{mL})$} \\
\hline & & Ceftazidime & $\begin{array}{l}\text { Ceftazidime } \\
\text {-avibactam }\end{array}$ & Cefotaxime & Aztreonam & $\begin{array}{l}\text { Piperacillin- } \\
\text { tazobactam }\end{array}$ & Imipenem & Meropenem \\
\hline $\mathrm{T}-970 / 19$ & $\begin{array}{l}\text { KPC-2, } \\
\text { VEB-25, } \\
\text { TEM-1, } \\
\text { OXA-10, } \\
\text { SHV-182 }\end{array}$ & $>256$ & 64 & 32 & $>256$ & $>256$ & $>32$ & $>32$ \\
\hline TrcT-970 & $\begin{array}{l}\text { VEB-25, } \\
\text { TEM-1, } \\
\text { OXA-10, }\end{array}$ & $>256$ & 32 & 4 & $>256$ & 16 & 0.25 & 0.032 \\
\hline$E-1037 / 19$ & $\begin{array}{l}\text { KPC-3, } \\
\text { VEB-25, } \\
\text { TEM-1, } \\
\text { OXA-10, } \\
\text { SHV-11 }\end{array}$ & $>256$ & $>256$ & 128 & $>256$ & $>256$ & $>32$ & $>32$ \\
\hline TrcE-1037 & $\begin{array}{l}\text { VEB-25, } \\
\text { TEM-1, } \\
\text { OXA-10, }\end{array}$ & $>256$ & 128 & 12 & $>256$ & 24 & 0.25 & 0.023 \\
\hline $\operatorname{TrcS}-2865$ & $\begin{array}{l}\text { VEB-1, } \\
\text { TEM-1, } \\
\text { OXA-10 }\end{array}$ & $>256$ & 0.25 & 4 & 128 & 2 & 0.19 & 0.023 \\
\hline 26R793 & None & 0.5 & 0.094 & 0.047 & 0.125 & 1 & 0.19 & 0.016 \\
\hline
\end{tabular}

KPC: Klebsiella pneumoniae carbapenemase; MIC: minimum inhibitory concentration; OXA: oxacillinase; SHV: sulfhydryl reagent variable $\beta$-lactamase; TEM: Temoniera $\beta$-lactamase; Trc: transconjugant; VEB: Vietnamese extended-spectrum $\beta$-lactamase.

T-970/19 and E-1037/19 are the VEB-producing Klebsiella pneumoniae clinical strains detected in this study. TrcT-970 and TrcE-1037 are the Escherichia coli transconjugant clones obtained by mating experiments using T-970/19 and E-1037/19 as donors and a $\beta$-lactamsusceptible $E$. coli K12 strain (26R793) as recipient.TrcS-2865 is a ceftazidime-avibactam-susceptible E. coli $26 R 793$ transconjugant containing bla ${ }_{\mathrm{VEB}-1}$, bla $\mathrm{OXA}-10$ and bla ${ }_{\mathrm{TEM}-1}$ that was used for control purposes.

Database (https://www.ncbi.nlm.nih.gov/bioproject/ PRJNA313047) of the National Center for Biotechnology Information (NCBI) as reference. The likely plasmid content was assessed with PlasmidFinder (http://cge. cbs.dtu.dk/services/PlasmidFinder/). T-970/19 and $\mathrm{E}-1037 / 19$ contained $b l a_{K \mathrm{PC}-2}$ and bla ${ }_{\mathrm{KPC}-3}$, respectively. Both strains harboured bla $a_{\mathrm{TEM}-1}$, bla ${ }_{\mathrm{OXA}-10}$ and a bla ${ }_{\mathrm{VEB}}{ }^{-}$ type gene which, compared with bla ${ }_{\text {VEB-1 }}$ (GenBank accession number: NG_050317) showed a non-synonymous mutation (A710G) leading to a novel Lys234Arg VEB mutant designated VEB-25 (GenBank accession number: MN862699). Notably, the Lys234Arg mutation has been observed in another VEB variant (VEB-20), which was reported in Pseudomonas aeruginosa from France (GenBank accession number: NG_063894), however the VEB-20 enzyme has an additional substitution compared to VEB-1 and VEB-25. Genes conferring resistance to aminoglycosides, fosfomycin, macrolides, phenicols, quinolones, rifampicin, sulfonamides, tetracycline and trimethoprim were also detected in both strains (Table 1). T-970/19 contained five replicons (IncA/C2, IncR, IncFIB(pKPHS1), IncFII(K), IncFIB(pQil)) while four replicons (IncA/C2, IncFIB(K), Inc $X_{3}$, ColRNAl) were identified in $\mathrm{E}-1037 / 19$.

\section{Transferability of ceftazidime-avibactam resistance and the role of the Vietnamese extended-spectrum $\beta$-lactamase-25}

Mating experiments using T-970/19 and E-1037/19 as donors and a $\beta$-lactam-susceptible Escherichia coli K12 strain (26R793) as recipient yielded CAZ-AVIresistant transconjugants (TrCT-970, TrcE-1037) at a frequency of $5 \times 10^{-6}$ per donor cell. Carriage of bla ${ }_{25}$, bla ${ }_{\text {OXA-10 }}$ and bla ${ }_{\text {TEM-1 }}$ by the transconjugant clones 
was confirmed by sequencing of PCR products. For control purposes, a CAZ-AVI-susceptible E. coli $26 \mathrm{R} 793$ transconjugant (TrCS-2865) containing bla ${ }_{\mathrm{VEB}-1}$, bla OXA ${ }_{10}$ and bla $a_{\text {TEM-1 }}$ was used. Production of the respective $\beta$-lactamases was confirmed by isoelectric focusing of bacterial cell extracts. The apparent isoelectric point of VEB-25 was $7 \cdot 5$.

TrcT-970 and TrcE-1037 were resistant to piperacillintazobactam, ceftazidime, ceftazidime-avibactam, cefotaxime and aztreonam yet remained susceptible to carbapenems according to the European Committee on Antimicrobial Susceptibility Testing (EUCAST) interpretation criteria [7]. The VEB-1-producing TrcS-2865 displayed resistance to ceftazidime, cefotaxime and aztreonam (Table 2). Since OXA-10 and TEM-1 do not hydrolyze ceftazidime while VEB-1 is a potent ceftazidimase [8], comparison of susceptibility data indicated that VEB-25 (i) inactivated ceftazidime and (ii) was not inhibited by avibactam. To test the latter hypothesis, the ability of avibactam to inhibit $\beta$-lactam hydrolysis by VEB-25 and VEB-1 was comparatively assessed. Experiments were carried out using cell extracts of TrcE-1037 and TrcS-2865 as sources of VEB enzymes and ceftazidime as a substrate. Inhibition caused by avibactam was measured by spectrophotometry as described previously [9]. Results, expressed as the concentration ( $\mathrm{nM}$ ) of inhibitor required to reduce hydrolysis rates by $50 \%\left(\mathrm{IC}_{50}\right)$, showed that VEB-25 preparations were considerably less inhibited by avibactam ( $\left.\mid C_{50}>1,000 \mathrm{nM}\right)$ as compared with those containing VEB-1 $\left(\mathrm{IC}_{50}=0.1 \mathrm{nM}\right)$.

\section{Structure of the Vietnamese extended- spectrum $\beta$-lactamase-25-encoding plasmids}

The sequences of bla ${ }_{\text {VEB-25}}$-carrying plasmids were determined after whole genome sequencing (WGS) of transconjugants TrcT-970 and TrcE-1037 following the bioinformatic subtraction of the DNA sequence of $E$. coli $26 \mathrm{R} 793$ (the complete sequences of Trc970-T and Trc1037-E have been deposited in GenBank under the accession numbers WUBHoooooooo and WUBG00000000). The size of pT-970 and pE-1037 plasmids were ca 170 and $150 \mathrm{kbp}$, respectively. Both plasmid sequence's scaffolds exhibited high similarity scores (>99\%) with previously published IncA/ C2 plasmids (GenBank accession number: CP027055) including the repA region, an intact conjugal transfer region and a parAB partitioning system. In pT-970 plasmid an additional IncR-derived segment including the repB replicase, a partitioning system and the vagCD and umuCD operons were identified. pT-970 and pE-1037 were punctuated by intact or truncated insertion sequences (IS) such as IS26, IS6100, IS1999, IS1, IS3-like, ISL3 and ISCR6. Both plasmids contained the

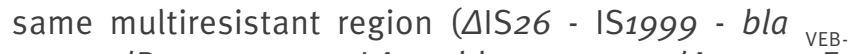
${ }_{25}$ - $a a d B$ - arr-2 - cmLA5 - bla oxA-10 - aadA1 - qacEdelta1 - $\triangle$ sul1 - floR2 - tetR - tet(G) - lysR - ISCR3-like - groEL - ISL3 - rmtB1 - bla TEM-1 $_{1}$ ).

\section{Discussion}

The presented findings clearly indicate that production of VEB-25 was responsible for resistance to CAZ-AVI. The enzyme was resistant to inhibition by $\mathrm{AVI}$ compared with its parental extended spectrum $\beta$-lactamase (ESBL) VEB-1. Moreover, it appears that VEB-25 retained the extended-spectrum properties of the VEB lineage. Similarly to VEB-25, substitution of the conserved lysine at Ambler's position 234 by arginine in laboratory and natural variants of SHV and KPC class A $\beta$-lactamases confers resistance to inactivation by $A V I$ and mechanism-based inhibitors [10-14]. It has been hypothesised that replacement of Lys234 by the longer side-chain Arg may alter the interaction with the adjacent Ser130 and consequently affect the catalytic process $[10,14]$. Yet, the mechanism remains unclear.

VEB-type $\beta$-lactamases are almost invariably associated with IncA/C2 replicons worldwide [15]. Enterobacteriaceae, mostly K. pneumoniae and Providencia stuartii, harbouring VEB-encoding multiresistant IncA/C2 plasmids have long been established in Greek hospitals occasionally causing outbreaks $[16,17]$. Indeed, during the course of this study $10 \%$ of the KPC-Kp were bla ${ }_{V E B}$-positive. Although the VEB-25 producers may have been selected by prior CAZ$\mathrm{AVI}$ use in the population, they were acquired during hospitalisation in two patients who had not received the drug. It is therefore reasonable to assume that these strains were already part of the nosocomial flora. Our sampling approach unfortunately, is unable to accurately estimate the extent of dissemination of VEB25 producers within the Greek Kp hospital populations. This is primarily due to the fact that the collection under investigation comprised only carbapenem-resistant isolates from either confirmed or suspected infections while neither carbapenem-susceptible nor surveillance culture isolates were included. Furthermore, participating hospitals are located solely in the Attika region. Whatever the case may be, the use of CAZ-AVI (introduced in this country in December 2017) will probably increase the clinical relevance of VEB-25-mediated resistance, given that the respective gene is carried by self-transferable plasmids that have been already acquired by high risk Kp clones potentially resulting in pandrug resistance.

\section{Acknowledgements}

We thank E. Siatravani for excellent technical assistance. We are also grateful to G.L. Daikos for helpful advice.

Funding statement

This research paper was made possible through the grant from the Stavros Niarchos Foundation to the Hellenic Pasteur Institute, as part of the Foundation's initiative to support the Greek research center ecosystem. 


\section{Conflict of interest}

None declared.

\section{Authors' contributions}

E. Voulgari: whole genome sequencing, plasmid characterisation. S.D. Kotsakis: data analysis. P. Giannopoulou: isolation and phenotypic characterisation of bacterial strains. E. Perivolioti: isolation and phenotypic characterisation of bacterial strains. L.S. Tzouvelekis: manuscript writing. V. Miriagou: enzyme characterisation, data analysis, manuscript writing.

\section{References}

1. Spiliopoulou I, Kazmierczak K, Stone GG. In vitro activity of ceftazidime/avibactam against isolates of carbapenem non-susceptible Enterobacteriaceae collected during the INFORM global surveillance programme (2015-17). J Antimicrob Chemother. 2019;dkz456. https://doi.org/10.1093/jac/dkz456 PMID: 31742604

2. Shields RK, Chen L, Cheng S, Chavda KD, Press EG, Snyder $A$, et al. Emergence of ceftazidime-avibactam resistance due to plasmid-borne blaKPC-3 mutations during treatment of carbapenem-resistant Klebsiella pneumoniae infections. Antimicrob Agents Chemother. 2017;61(3):e02097-16. https:// doi.org/10.1128/AAC.02097-16 PMID: 28031201

3. Haidar G, Clancy CJ, Shields RK, Hao B, Cheng S, Nguyen MH. Mutations in blaKPC-3 that confer ceftazidime-avibactam resistance encode novel KPC-3 variants that function as extended-spectrum $\beta$-lactamases. Antimicrob Agents Chemother. 2017;61(5):e02534-16. https://doi.org/10.1128/ AAC.02534-16 PMID: 28223379

4. Gaibani P, Campoli C, Lewis RE, Volpe SL, Scaltriti E, Giannella $M$, et al. In vivo evolution of resistant subpopulations of KPC-producing Klebsiella pneumoniae during ceftazidime/ avibactam treatment. J Antimicrob Chemother. 2018;73(6):1525 9. https://doi.org/10.1093/jac/dkyo82 PMID: 29566151

5. Räisänen K, Koivula I, Ilmavirta H, Puranen S, Kallonen T, Lyytikäinen 0 , et al. Emergence of ceftazidime-avibactamresistant Klebsiella pneumoniae during treatment, Finland, December 2018. Euro Surveill. 2019;24(19):1900256. https:// doi.org/10.2807/1560-7917.ES.2019.24.19.1900256 PMID: 31088601

6. European Centre for Disease Prevention and Control (ECDC). Emergence of resistance to ceftazidime-avibactam in carbapenem-resistant Enterobacteriaceae - 12 June 2018. Stockholm; ECDC; 2018. Available from: https://ecdc.europa. eu/en/publications-data/rapid-risk-assessment-emergenceresistance-ceftazidime-avibactam-carbapenem

7. The European Committee on Antimicrobial Susceptibility Testing (EUCAST). Breakpoint tables for interpretation of MICs and zone diameters. Version 9.0. Växjö: EUCAST; 2019. Available from; http://www.eucast.org.

8. Poirel L, Naas T, Guibert M, Chaibi EB, Labia R, Nordmann P. Molecular and biochemical characterization of VEB-1, a novel class $A$ extended-spectrum $\beta$-lactamase encoded by an Escherichia coli integron gene. Antimicrob Agents Chemother. 1999;43(3):573-81. https://doi.org/10.1128/AAC.43.3.573 PMID: 10049269

9. Prinarakis EE, Miriagou V, Tzelepi E, Gazouli M, Tzouvelekis LS. Emergence of an inhibitor-resistant $\beta$-lactamase (SHV-10) derived from an SHV- 5 variant. Antimicrob Agents Chemother. 1997;41(4):838-40. https://doi.org/10.1128/AAC.41.4.838 PMID: 9087500

10. Mendonça N, Manageiro V, Robin F, Salgado MJ, Ferreira E, Caniça M, et al. The Lys234Arg substitution in the enzyme SHV72 is a determinant for resistance to clavulanic acid inhibition. Antimicrob Agents Chemother. 2008;52(5):1806-11. https:// doi.org/10.1128/AAC.01381-07 PMID: 18316518

11. Dubois V, Poirel L, Demarthe F, Arpin C, Coulange L, Minarini LA, et al. Molecular and biochemical characterization of SHV56, a novel inhibitor-resistant $\beta$-lactamase from Klebsiella pneumoniae. Antimicrob Agents Chemother. 2008;52(10):37924. https://doi.org/10.1128/AAC.00387-08 PMID: 18663019

12. Manageiro V, Ferreira E, Albuquerque L, Bonnet R, Caniça M Biochemical study of a new inhibitor-resistant $\beta$-lactamase, SHV-84, produced by a clinical Escherichia coli strain. Antimicrob Agents Chemother. 2010;54(5):2271-2. https://doi. org/10.1128/AAC.01442-09 PMID: 20211886
13. Winkler ML, Papp-Wallace KM, Taracila MA, Bonomo RA Avibactam and inhibitor-resistant SHV $\beta$-lactamases. Antimicrob Agents Chemother. 2015;59(7):3700-9. https://doi. org/10.1128/AAC.04405-14 PMID: 25691639

14. Papp-Wallace KM, Winkler ML, Taracila MA, Bonomo RA. Variants of $\beta$-lactamase KPC-2 that are resistant to inhibition by avibactam. Antimicrob Agents Chemother. 2015;59(7):37107. https://doi.org/10.1128/AAC.04406-14 PMID: 25666153

15. Poirel L, Villa L, Bertini A, Pitout JD, Nordmann P, Carattoli A. Expanded-spectrum $\beta$-lactamase and plasmid-mediated quinolone resistance. Emerg Infect Dis. 2007;13(5):803-5. https://doi.org/10.3201/eid1305.061293 PMID: 18044054

16. Papagiannitsis CC, Tryfinopoulou K, Giakkoupi P, Pappa O, Polemis M, Tzelepi E, et al. Carbapenemase Study Group. Diversity of acquired $\beta$-lactamases amongst Klebsiella pneumoniae in Greek hospitals. Int J Antimicrob Agents. 2012;39(2):178-80. https://doi.org/10.1016/j. ijantimicag.2011.09.024 PMID: 22104281

17. Giakkoupi P, Tryfinopoulou K, Polemis M, Pappa O, Miriagou $\mathrm{V}$, Vatopoulos A. Circulation of a multiresistant, conjugative, IncA/C plasmid within the nosocomial Providencia stuartii population in the Athens area. Diagn Microbiol Infect Dis. 2015;82(1):62-4. https://doi.org/10.1016/j. diagmicrobio.2015.02.009 PMID: 25752202

\section{License, supplementary material and copyright}

This is an open-access article distributed under the terms of the Creative Commons Attribution (CC BY 4.0) Licence. You may share and adapt the material, but must give appropriate credit to the source, provide a link to the licence and indicate if changes were made.

Any supplementary material referenced in the article can be found in the online version.

This article is copyright of the authors or their affiliated institutions, 2020. 